\title{
Fibroblast Growth Factor-23 (FGF-23) e Sindrome Cardiorenale
}

\author{
Luca Di Lullo ${ }^{1}$, Rodolfo Rivera ${ }^{2}$, Antonio De Pascalis ${ }^{3}$, Fulvio Floccari ${ }^{4}$, Vincenzo Barbera ${ }^{1}$, Claudio Ronco ${ }^{5}$, Alberto Santoboni ${ }^{1}$ \\ ${ }^{1}$ U.O.C. Nefrologia e Dialisi, Ospedale "L. Parodi-Delfino", Colleferro (RM) \\ ${ }^{2}$ Divisione di Nefrologia, Ospedale S. Gerardo, Monza \\ ${ }^{3}$ U.O.C. Nefrologia, Dialisi e Trapianto, Ospedale "V. Fazzi", Lecce \\ "U.O.C. Nefrologia e Dialisi, Ospedale "S. Paolo", Civitavecchia (RM) \\ 5International Renal Research Institute, Ospedale "S. Bortolo", Vicenza
}

\begin{abstract}
Fibroblast Growth Factor - 23 (FGF-23) e Cardiorenal Syndrome
Several abnormalities in chronic kidney disease-related mineral and bone disease (CKD-MBD) have emerged as novel risk factors in excess cardiovascular mortality in patients with CKD and end-stage renal disease (ESRD). Hyperphosphatemia, vascular calcifications development, and decreased active vitamin D production, leading to activation of the renin angiotensin system (RAS), have been identified as the primary cause of CKD-MBDassociated mortality in CKD.

Recently, the fibroblast growth factor-23 (FGF-23), a newly discovered hormone produced in the bone that regulates phosphate and vitamin D metabolism by the kidney, has been reported as a strong predictor of adverse cardiovascular outcomes in patients with CKD and ESRD.

The main physiological functions of FGF-23 are mediated by the activation of the FGF receptor/ $\alpha$-klotho co-receptor complexes in target tissues. Elevated FGF-23 has been associated with left ventricular hypertrophy (LVH), and it has been suggested that FGF-23 may induce myocardial hypertrophy through a direct effect on cardiac myocytes.

Understanding of FGF-23's pathophysiology and mechanisms of action responsible for its negative effects will be necessary to develop therapeutic strategies to treat CKD-MBD.
\end{abstract}

Keywords: FGF-23, Chronic kidney disease, Cardiovascular disease, Sevelamer Carbonate, Lanthanum carbonate

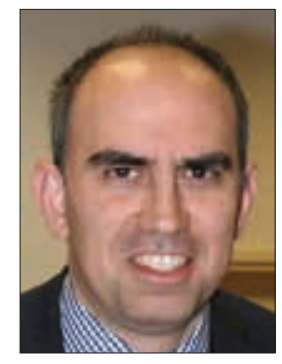

\section{Introduzione}

La patologia cardiovascolare è ormai universalmente riconosciuta come la principale causa di mortalità e morbidità nei pazienti affetti da malattia renale cronica (1). Dalla letteratura scientifica e dai grandi trial clinici effettuati nel corso degli ultimi anni è emerso come diverLuca Di Lullo si fattori di rischio, tradizionali (diabete mellito, ipertensione arteriosa, età, ses-

Accepted: February 12, 2015

Published online: March 2, 2015

Indirizzo per la corrispondenza:

Dr. Luca Di Lullo

U.O.C. Nefrologia e Dialisi, Ospedale "L. Parodi-Delfino"

Piazza A. Moro 1

00034 Colleferro (RM)

dilulloluca69@gmail.com so, abitudini voluttuarie) e non, concorrano alla maggiore incidenza di eventi cardiovascolari nei pazienti nefropatici.

Nella popolazione affetta da malattia renale cronica è paradossale osservare come i fattori di rischio cardiovascolare tradizionale siano associati a outcome ben più favorevoli rispetto alla presenza di fattori di rischio più specificamente legati alla stessa CKD: anemia, stato infiammatorio cronico e iperparatiroidismo secondario sono alcuni dei nuovi attori comparsi sulla scena del rischio cardiovascolare in questa particolare coorte di pazienti.

La discussione che seguirà si concentrerà, in modo particolare, sullo stretto legame esistente tra malattia renale cronica, patologia ossea-alterazioni del metabolismo minerale (CKD-MBD) ed eccesso di mortalità per cause cardiovascolari.

II link fisiopatologico tra alterazioni del metabolismo minerale/osseo e aumento del rischio cardiovascolare non è tuttora del tutto chiarito, anche se sono in fase di studio diverse ipotesi in merito.

Nell'ambito della CKD-MBD, l'iperfosforemia, l'ipo-ipercalcemia o l'ipo-iperparatiroidismo (iperPTH) rappresentano 
singolarmente $o$ in associazione tutti potenziali fattori favorenti la progressione di un danno cardiovascolare.

Negli ultimi tempi, inoltre, diversi nuovi attori si sono affacciati sulla scena e, in particolare, una molecola, tra le altre, sembra essere quella maggiormente indiziata per il suo contributo alla progressione della patologia cardiovascolare: il FGF (fibroblast growth factor)-23 (2).

FGF-23 è, in primis, un regolatore dei livelli di fosforemia per poi contribuire all'omeostasi della vitamina $\mathrm{D}$; a livello renale favorisce l'eliminazione di fosfato inibendone il riassorbimento a livello del tubulo prossimale (determinando, quindi, un aumento della fosfaturia). Allo stesso tempo, FGF-23 incide negativamente sui livelli circolanti di $1.25(\mathrm{OH})$, vitamina $\mathrm{D}(2,3)$.

\section{Ruolo e regolazione dei livelli circolanti di FGF-23 in condizioni di normalità}

FGF-23 è una molecola proteica di $32 \mathrm{kDa}$ prodotta, a livello osseo, da osteoblasti e osteociti e appartiene alla famiglia dei fattori di crescita dei fibroblasti e, più in particolare, al sottogruppo FGF 19, che si differenzia per la presenza di una sequenza aminoacidica peculiare a livello della regione C-terminale. Grazie a tale caratteristica di natura molecolare, i membri di questo sottogruppo di FGF non vengono inglobati nel contesto della matrice extracellulare, ma rilasciati in circolo con le stesse modalità di un ormone circolante (4).

Per l'attivazione dello specifico recettore e affinché FGF-23 possa esercitare il suo effetto sui tessuti bersaglio, è indispensabile la presenza di un cofattore, $\alpha$-klotho, in grado di legarsi al frammento C-terminale di FGF-23 (5). $\alpha$-klotho è espresso a livello di diversi organi e tessuti, in particolar modo a livello delle cellule dei tubuli distali e dei collettori renali, dei plessi coroidei cerebrali, delle paratiroidi e dell'ipofisi e, in misura minore, a livello delle cellule del nodo seno-atriale, della placenta, del muscolo scheletrico, della vescica, del pancreas, del testicolo e del colon $(6,7)$.

Come in parte già anticipato, il bersaglio principale di FGF-23 è il complesso recettore FGF-klotho a livello renale, la cui attivazione comporta l'inibizione del riassorbimento di fosfato in sede tubulare prossimale; inoltre, l'attivazione del complesso recettoriale comporta la soppressione dei livelli circolanti di $1.25(\mathrm{OH})_{2}$ mediante un doppio meccanismo d'azione: inibizione dell'attività $1 \alpha$ idrossilasi e attivazione della 24 idrossilasi (3).

Contestualmente all'effetto esercitato sui livelli circolanti di vitamina D attiva, l'azione di FGF-23 si esplica mediante l'attività inibente la secrezione di PTH, così come evidenziato da studi in vivo e in vitro (8). Nonostante queste ultime osservazioni, nella pratica clinica l'aumento dei livelli sierici di FGF-23 si associa a un contestuale aumento dei livelli di PTH e a un quadro di iperparatiroidismo secondario, spesso refrattario alla terapia convenzionale (9). Tale apparente incongruenza può essere spiegata dal fatto che, in corso di uremia, la ghiandola paratiroidea risulta insensibile all'azione esercitata da FGF-23 a causa della verosimile ridotta espressione di klotho (10).

Infatti, oltre ai già menzionati effetti sui livelli di fosforo, vitamina D e PTH, FGF-23 è in grado di sopprimere la trascrizione, a livello renale, dei geni per il klotho $(3,11)$.

II principale regolatore dei livelli di FGF-23 resta I'1.25 $(\mathrm{OH})_{2}$ vitamina $\mathrm{D}$ in grado di stimolare la produzione di FGF-23 e, soprattutto, di mantenere un circuito di feedback con FGF-23 stesso (12).

L'eventuale ruolo di altre molecole regolatrici della sintesi di FGF-23 resta tuttora in forte dubbio; è stato suggerito come il PTH stesso sia in grado di incrementare la sintesi di FGF-23 (13), ma tale evidenza non è stata confermata da tutti gli studi condotti in tal senso (14).

Evidenze più recenti suggeriscono come un effetto diretto sulla sintesi di FGF-23 possa essere collegato ai processi di mineralizzazione e di rimodellamento osseo attraverso dei meccanismi, ancora poco chiari, di attivazione recettoriale in organi bersaglio diversi da quelli al momento conosciuti (15).

Certamente i livelli di FGF-23 sono influenzati da quelli di leptina, estrogeni e glucocorticoidi (2).

Quello che sembra essere particolarmente intrigante è che i livelli di fosforemia non sembrano esercitare effetti drammatici sulla produzione di FGF-23, a testimonianza del fatto che gli effetti della fosforemia sui livelli di FGF-23 potrebbero essere mediati da altre molecole in grado di influenzare il processo di rimodellamento osseo $(16,17)$.

\section{II ruolo di FGF-23 in corso di malattia renale cronica (CKD)}

I livelli sierici di FGF-23 aumentano sin dai primi stadi di CKD e rappresentano la prima spia delle alterazioni del metabolismo minerale $(18,19)$.

Strettamente collegato alla riduzione del filtrato glomerulare (GFR), si assiste all'aumento dei livelli sierici di FGF-23, PTH e fosforo con una concomitante diminuzione dei livelli sierici di vitamina $D$ attiva (18-20); in particolar modo, i livelli sierici di FGF-23 possono aumentare anche fino a 1000 volte rispetto a quelli documentabili nei pazienti sani.

Allo stato attuale non è identificabile una ben precisa motivazione per la quale i livelli di FGF-23 aumentano in maniera così evidente in corso di CKD.

Uno dei motivi può essere ricercato nella stimolazione abnorme alla secrezione di FGF-23 esercitata da sostanze liberate dal parenchima renale danneggiato o negli effetti esercitati dallo stato uremico sui processi di mineralizzazione ossea. Altre evidenze supportano il ruolo cruciale del fosforo nella stimolazione incontrollata della secrezione di FGF-23 (21).

Negli stadi avanzati di CKD (con eGFR $<25 \mathrm{~mL} / \mathrm{min} / 1.73 \mathrm{~m}^{2}$ ), anche i livelli elevati di PTH esercitano un effetto favorevole per l'aumento di FGF-23, come testimonia la riduzione dei li- 
velli di FGF-23 dopo paratiroidectomia (22). Sempre in corso di CKD, un aumento dei livelli di FGF-23 circolante è favorito dalla terapia con analoghi della vitamina D attiva (23).

Come in parte già anticipato, appare inaspettatamente marginale il ruolo della fosforemia nella regolazione dei livelli di FGF-23. Evidenze piuttosto recenti hanno documentato come una dieta contenente $900 \mathrm{mg}$ di fosforo giornaliero associata alla somministrazione di un chelante del fosforo sia in grado di ottenere una riduzione pari al 35\% dei livelli sierici di FGF-23, laddove la sola dieta o il solo impiego del chelante non sono stati in grado di influenzare le concentrazioni di FGF-23 circolante (24).

Studi condotti in animali da laboratorio hanno permesso di formulare l'ipotesi che l'aumento dei livelli di FGF-23 rappresenta probabilmente un meccanismo di compenso in grado di mantenere una concentrazione neutrale di fosforo in condizioni di ridotta riserva funzionale renale (25).

Se finora l'aumento dei livelli di FGF-23 è stato interpretato come un meccanismo di compenso e di adattamento al nuovo equilibrio metabolico venutosi a creare in corso di CKD, dall'altro lato livelli estremi di aumento dei tassi circolanti di FGF-23 possono essere visti come parte di un processo di cattivo adattamento al nuovo scenario di rimodellamento osseo. Numerosi studi osservazionali hanno evidenziato come elevati livelli sierici di FGF-23 si accompagnino a un aumento del tasso di mortalità nei pazienti con malattia renale cronica terminale (ESRD, end stage renal disease) ma non sottoposti a trattamento dialitico e nei pazienti portatori di trapianto renale, indipendentemente dalla presenza di iperfosforemia e/o iperparatiroidismo $(26,27)$.

L'aumento del tasso di mortalità associato all'aumento dei livelli sierici di FGF-23 è verosimilmente dovuto al contestuale aumento della patologia cardiovascolare come suggerito da diversi lavori scientifici che hanno messo in correlazione elevati livelli sierici di FGF-23, presenza di calcificazioni cardiache, ipertrofia ventricolare sinistra, disfunzione endoteliale e aumentati livelli sierici di citochine ad azione pro-infiammatoria (28-32) (Fig. 1).

\section{FGF-23 e outcome cardiovascolari (e non): meccanismi fisiopatologici}

Quanto descritto nella parte finale del precedente paragrafo ci porta a concludere come la maggior parte degli eventi sfavorevoli legati all'aumento dei livelli sierici di FGF-23 abbia come bersaglio principale l'apparato cardiovascolare. La presenza di anomalie biochimiche correlate a un aumento della mortalità cardiovascolare come iperparatiroidismo, iperfosforemia e ipovitaminosi $D$, correlate con aumentati livelli di FGF-23, permette di postulare come l'aumento delle concentrazioni sieriche di FGF-23 possa contribuire all'aumentata incidenza di patologie cardiovascolari nei pazienti affetti da CKD.

Per spiegare l'effetto negativo esercitato da elevati livelli

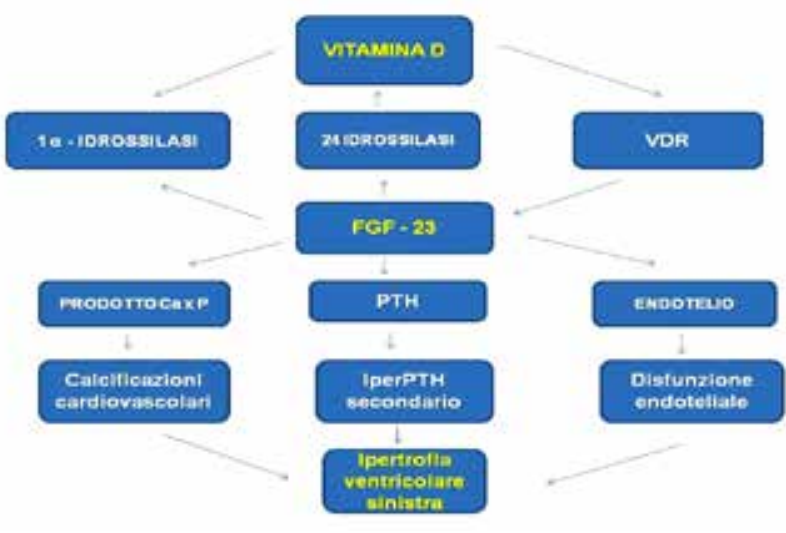

Fig. 1 - FGF-23 e genesi del danno cardiovascolare.

sierici di FGF-23 a livello cardiaco, bisogna tornare ai rapporti esistenti tra FGF-23 e klotho. In particolar modo va considerato come elevati livelli di FGF-23 possano rappresentare una spia della riduzione di attività di klotho il quale esercita un'attività ormonale di tipo paracrino. Livelli elevati di FGF-23, infatti, si accompagnano a una ridotta produzione di klotho con effetti sfavorevoli a livello cardiaco, in quanto klotho esercita normalmente un'azione cardioprotettiva mediante la down regulation dei canali TRPV5 (Transient Receptor Potential Vanilloid-5); la diminuzione dei livelli di klotho contribuisce, inoltre, all'instaurarsi di un quadro di ipertrofia ventricolare sinistra (LVH), evento frequente e temibile nei pazienti affetti da CKD (33).

FGF-23, inoltre, può essere coinvolto nella genesi delle comorbidità cardiovascolari indipendentemente dal coinvolgimento di altri "attori" più implicati nella patogenesi della malattia ossea correlata a CKD (CKD-MBD: chronic kidney disease-mineral bone disease). Diversi studi osservazionali hanno supportato questa ipotesi e, tra l'altro, il livello di incremento delle concentrazioni sieriche di FGF-23 nella malattia renale cronica allo stadio terminale supera di gran lunga gli incrementi di altre molecole implicate nella genesi del danno cardiovascolare in corso di CKD-MBD (PTH, calcio, fosforo).

Tra l'altro, altre evidenze scientifiche supportano un dato che non va assolutamente sottovalutato: elevati livelli di FGF-23 si associano a un aumento della mortalità anche in pazienti che non presentano alterazioni della funzione renale, né, tantomeno, alterazioni biochimiche e sintomi clinici indicativi di patologia ossea (34).

Infine, dati estremamente recenti sul ruolo fisiologico e fisiopatologico di FGF-23 indicano che tale molecola può esercitare effetti diretti o indiretti sull'apparato cardiovascolare con modalità totalmente distinte rispetto agli effetti esplicati a livello del metabolismo minerale/osseo.

A quest'ultimo proposito c'è da registrare l'interessante "connivenza" tra FGF-23 e sistema renina-angiotensina (RAS). II RAS, come ben documentato, gioca un ruolo preminente 
a livello di morbidità e mortalità cardiovascolare, causando ipertensione arteriosa, disfunzione barocettoriale, attivazione simpatica, progressione del danno aterosclerotico, disfunzione endoteliale e inibizione della fibrinolisi con evoluzione più o meno rapida verso quadri significativi di fibrosi miocardica, ipertrofia ventricolare sinistra e insufficienza cardiaca (35).

FGF-23 è in grado di stimolare l'attività del RAS sopprimendo, a livello renale, l'espressione dei geni che codificano per ACE-2 (angiotensin converting enzyme-2), indipendentemente dalle altre alterazioni biochimiche tipiche della CKD-MBD (36).

L'attivazione del RAS potrebbe anche spiegare il perché dello stretto legame esistente tra elevati livelli di FGF-23 e outcome cardiovascolari sfavorevoli, come, per esempio, l'ipertrofia ventricolare sinistra e la fibrosi miocardica (36).

Una seconda potenziale modalità con la quale FGF-23 può causare danni cardiovascolari risiede probabilmente nel ruolo giocato nei processi infiammatori cronici. Evidenze sperimentali suggeriscono come FGF-23 sia in grado di stimolare la produzione di citochine ad azione pro-infiammatoria come lipocalina-2, TGF- $\beta$ (transforming growth factor- $\beta$ ) e TNF- $\alpha$ (tumor necrosis factor- $\alpha$ ) (36); non sono, al momento, del tutto chiariti i meccanismi molecolari grazie ai quali avviene quanto appena descritto.

Negli ultimi anni è stata proposta una teoria eziopatogenetica piuttosto intrigante secondo la quale non solo FGF-23 potrebbe essere coinvolto nella genesi del danno cardiovascolare indipendentemente dal suo ruolo in corso di CKD-MBD, ma tali azioni si esplicherebbero direttamente su tutti quegli organi e tessuti che non sono in grado di esprimere $\alpha$-klotho.

Come già abbondantemente discusso, FGF-23 è in grado di promuovere lo sviluppo di ipertrofia ventricolare sinistra, come evidenziato da studi eseguiti in vitro e su animali da esperimento (31). Le cellule miocardiche non esprimono klotho, a testimonianza del fatto che, probabilmente, FGF-23 non necessita di klotho per legarsi ai suoi recettori di membrana in questa sede.

Ciò che va confermato è se tali effetti esercitati da FGF-23 siano possibili in caso di concentrazioni molto elevate come quelle documentate in corso di ESRD; quanto poco prima descritto sulla presenza di elevati livelli di FGF-23 e sulla presenza di ipertrofia ventricolare sinistra in pazienti con funzione renale normale suggerisce che la sola presenza di elevate concentrazioni di FGF-23 non può spiegare gli effetti cardiovascolari dello stesso FGF-23 ma che, verosimilmente, altri mediatori entrano in gioco nel determinare quei quadri, spesso imponenti, di fibrosi miocardica, documentati in pazienti affetti da malattia renale cronica terminale.

\section{Strategie terapeutiche}

Determinare con certezza se FGF-23 sia in grado di esercitare effetti diretti o indiretti a livello dell'apparato cardiovascolare e se tali effetti rientrino nello spettro più ampio della CKD-MBD o ne siano completamente slegati diventa cruciale nel momento in cui si devono studiare adeguate strategie terapeutiche.

Tali strategie non possono prescindere dalla correzione di tutte quelle alterazioni del profilo biochimico tipiche della CKD-MBD come iperfosforemia, iperparatiroidismo e, soprattutto, deficit di vitamina D.

Allo stato attuale è anche vero che nessun trial clinico ha chiarito in maniera definitiva come la correzione di tali squilibri metabolici possa incidere in modo favorevole sugli outcome cardiovascolari in corso di CKD-MBD, rendendo oltremodo complicata la gestione del paziente che presenta elevati livelli sierici di FGF-23.

D’altro canto si può tranquillamente affermare che gli interventi terapeutici attualmente impiegati per il controllo della CKD-MBD incidono anche sui livelli circolanti di FGF-23.

La somministrazione prolungata di chelanti del fosforo privi di calcio, come il sevelamer e il lantanio carbonato, si è dimostrata in grado di ottenere una riduzione significativa dei livelli plasmatici di FGF-23 $(37,38)$ a testimonianza del fatto che gli effetti farmacologici esercitati dai chelanti non si esplicano solo sui livelli di fosforo ma che, probabilmente, entrano in gioco anche diversi altri fattori eziopatogenetici (come, per esempio, il rimodellamento osseo); a supporto di tale osservazione ci sono dati che hanno evidenziato come la somministrazione per brevi periodi di lantanio carbonato non fosse in grado di ridurre i livelli di FGF-23, nonostante una concomitante significativa riduzione della fosfaturia (16).

Anche i farmaci impiegati nel trattamento dell'iperparatiroidismo secondario sono in grado di agire sulle concentrazioni plasmatiche di FGF-23; la somministrazione di vitamina $D$ attiva è in grado di stimolare la produzione di FGF-23, determinando un aumento dei livelli sierici (39), mentre la terapia a base di cinacalcet sembra essere in grado di ridurre la concentrazione plasmatica di FGF-23 $(39,40)$.

L'effetto contrastante esercitato dalla terapia con vitamina D attiva o con cinacalcet ci pone l'interrogativo sul significato clinico di tali risultati. La terapia con vitamina $D$ attiva è stata associata, nei pazienti affetti da ESRD, a una diminuzione della mortalità cardiovascolare in numerosi studi osservazionali, ma mancano ancora dati provenienti da trial clinici controllati. Una possibile spiegazione del perché la somministrazione di vitamina $D$ attiva comporti un aumento dei livelli di FGF-23 risiederebbe nel fatto che FGF-23 è in grado di determinare una diminuzione dei livelli di $1.25(\mathrm{OH})$, vitamina $\mathrm{D}$. La somministrazione di vitamina $D$ attiva potrebbe, quindi, antagonizzare l'effetto diretto esercitato da FGF-23 sull'1.25 $(\mathrm{OH})_{2}$ vitamina D.

La terapia con cinacalcet sembra esercitare effetti opposti rispetto a quelli promossi dalla vitamina $D$ attiva e il principale vantaggio risiede nel fatto che cinacalcet, oltre a favorire la riduzione dei livelli di FGF-23, non determina un aumento di quelli di calcio e fosforo sierico. Tutto questo si tradurrebbe nella possibilità di ottenere outcome più favorevoli rispetto alla terapia con vitamina D attiva; nella pratica clinica, però, le cose sembrano andare diversamente, come evidenziato dai 
risultati di un trial abbastanza recente il quale, confrontando le due strategie terapeutiche (cinacalcet vs vitamina $D$ attiva), non ha evidenziato una significativa riduzione della mortalità per cause cardiovascolari nel gruppo di pazienti trattato con cinacalcet (41).

Alla luce delle già citate interazioni FGF-23-RAS e dell'attività pro-infiammatoria di FGF-23, ulteriori possibilità terapeutiche potrebbero essere offerte dall'impiego di inibitori dell'ACE-2 (ACE inibitori, antagonisti recettoriali dell'angiotensina, antagonisti recettoriali dell'aldosterone) ovvero da farmaci in grado di influenzare lo stato di infiammazione cronica dei pazienti affetti da CKD.

Allo stesso tempo, inoltre, alla luce del fatto che FGF-23 può esercitare effetti diretti a livello delle cellule miocardiche, sarebbe almeno indicato poter agire a livello di organi bersaglio prevenendo gli effetti lesivi causati dall'eccesso dei livelli di FGF-23 ma, anche in questo caso, siamo lontani dall'aver trovato delle ricette miracolose. Un primo tentativo è stato fatto in studi condotti in pazienti affetti da rachitismo $X$-linked e CKD $(42,43)$ allo scopo di trattare le alterazioni biochimiche e del turnover osseo causate dall'eccesso di FGF-23. La terapia praticata ha comportato l'insorgenza di ipofosforemia, un incremento significativo delle calcificazioni aortiche e un aumento della mortalità; se ne conclude che FGF-23, probabilmente, si trova al centro di una serie di circuiti di feedback e l'azzeramento (o la notevole riduzione) dei suoi livelli plasmatici può risultare persino deleterio.

\section{Conclusioni}

FGF-23 si è chiaramente connotato come un nuovo e potente fattore di rischio di mortalità cardiovascolare in pazienti affetti da CKD, soprattutto in corso di ESRD.

È sicuramente coinvolto in diversi processi fisiologici indipendenti dal suo ruolo principe di regolatore dell'omeostasi ossea e minerale; presenta, inoltre, una forte associazione con gli outcome sfavorevoli e con la mortalità cardiovascolare, indipendentemente dalla presenza di una condizione di CKD-MBD.

La delucidazione delle funzioni fisiologiche e l'impatto in diverse condizioni patologiche di FGF-23 diventa, al contempo, una conditio sine qua non per la messa a punto di nuove e più definitive strategie terapeutiche e il conseguimento di outcome (cardiovascolari e non) decisamente più favorevoli nei pazienti affetti da CKD ed ESRD.

\section{Disclosures}

Financial support: No financial support was received for this submission.

Conflict of interest: The authors have no conflict of interest.

\section{Bibliografia}

1. Kalantar-Zadeh K, Shah A, Duong U, Hechter RC, Dukkipati R, Kovesdy CP. Kidney bone disease and mortality in CKD: revisi- ting the role of vitamin $D$, calcimimetics, alkaline phosphatase, and minerals. Kidney Int Suppl 2010;117:S10-21.

2. Martin A, David V, Quarles LD. Regulation and function of the FGF23/klotho endocrine pathways. Physiol Rev 2012;92: 131-55.

3. Quarles LD. Role of FGF23 in vitamin D and phosphate metabolism: implications in chronic kidney disease. Exp Cell Res 2012;318:1040-8.

4. Goetz R, Nakada Y, Hu MC, et al. Isolated C-terminal tail of FGF23 alleviates hypophosphatemia by inhibiting FGF23-FGFR-Klotho complex formation. Proc Natl Acad Sci USA 2010;107:407-12.

5. Zhang X, Ibrahimi OA, Olsen SK, Umemori H, Mohammadi M, Ornitz DM. Receptor specificity of the fibroblast growth factor family. The complete mammalian FGF family. J Biol Chem 2006; 281:15694-700.

6. Zhou L, Li Y, Zhou D, Tan RJ, Liu Y. Loss of Klotho contributes to kidney injury by derepression of Wnt/ $\beta$-catenin signaling. J Am Soc Nephrol 2013;24:771-85.

7. Lindberg K, Olauson $\mathrm{H}, \mathrm{Amin} \mathrm{R}$, et al. Arterial Klotho expression and FGF23 effects on vascular calcification and function. PLoS One 2013;8:e60658.

8. Ben-Dov IZ, Galitzer H, Lavi-Moshayoff V, et al. The parathyroid is a target organ for FGF23 in rats. J Clin Invest 2007;117:4003-8.

9. Nakanishi S, Kazama JJ, Nii-Kono T, et al. Serum fibroblast growth factor-23 levels predict the future refractory hyperparathyroidism in dialysis patients. Kidney Int 2005;67:1171-8.

10. Galitzer H, Ben-Dov IZ, Silver J, Naveh-Many T. Parathyroid cell resistance to fibroblast growth factor 23 in secondary hyperparathyroidism of chronic kidney disease. Kidney Int 2010;77:211-8.

11. Stubbs JR, Liu S, Tang W, et al. Role of hyperphosphatemia and 1,25-dihydroxyvitamin $D$ in vascular calcification and mortality in fibroblastic growth factor 23 null mice. J Am Soc Nephrol 2007; 18:2116-24.

12. Liu S, Tang W, Zhou J, et al. Fibroblast growth factor 23 is a counterregulatory phosphaturic hormone for vitamin D. J Am Soc Nephrol 2006;17:1305-15.

13. Rhee $\mathrm{Y}$, Allen MR, Condon $\mathrm{K}$, et al. PTH receptor signaling in osteocytes governs periosteal bone formation and intracortical remodeling. J Bone Miner Res 2011;26:1035-46.

14. Saji F, Shigematsu T, Sakaguchi T, et al. Fibroblast growth factor 23 production in bone is directly regulated by $1 \alpha, 25$ dihydroxyvitamin D, but not PTH. Am J Physiol Renal Physiol 2010;299:F1212-7.

15. Martin A, Liu S, David V, et al. Bone proteins PHEX and DMP1 regulate fibroblastic growth factor Fgf23 expression in osteocytes through a common pathway involving FGF receptor (FGFR) signaling. FASEB J 2011;25:2551-62.

16. Isakova T, Gutierrez OM, Smith K, et al. Pilot study of dietary phosphorus restriction and phosphorus binders to target fibroblast growth factor 23 in patients with chronic kidney disease. Nephrol Dial Transplant 2011;26:584-91.

17. Vervloet MG, van Ittersum FJ, Buttler RM, Heijboer AC, Blankenstein MA, ter Wee PM. Effects of dietary phosphate and calcium intake on fibroblast growth factor-23. Clin J Am Soc Nephrol 2011;6:383-9.

18. Stubbs JR, He N, Idiculla A, et al. Longitudinal evaluation of FGF23 changes and mineral metabolism abnormalities in a mouse model of chronic kidney disease. J Bone Miner Res 2012;27:38-46.

19. Isakova T, Wahl P, Vargas GS, et al. Fibroblast growth factor 23 is elevated before parathyroid hormone and phosphate in chronic kidney disease. Kidney Int 2011;79:1370-8.

20. Gutierrez $\mathrm{O}$, Isakova $\mathrm{T}$, Rhee $\mathrm{E}$, et al. Fibroblast growth factor-23 mitigates hyperphosphatemia but accentuates calcitriol defi- 
ciency in chronic kidney disease. J Am Soc Nephrol 2005;16: 2205-15.

21. Shimada T, Urakawa I, Isakova T, et al. Circulating fibroblast growth factor 23 in patients with end-stage renal disease treated by peritoneal dialysis is intact and biologically active. J Clin Endocrinol Metab 2010;95:578-85.

22. Sato $\mathrm{T}$, Tominaga $\mathrm{Y}$, Ueki $\mathrm{T}$, et al. Total parathyroidectomy reduces elevated circulating fibroblast growth factor 23 in advanced secondary hyperparathyroidism. Am J Kidney Dis 2004;44:481-7.

23. Stubbs JR, Idiculla A, Slusser J, Menard R, Quarles LD. Cholecalciferol supplementation alters calcitriol-responsive monocyte proteins and decreases inflammatory cytokines in ESRD. J Am Soc Nephrol 2010;21:353-61.

24. Isakova T, Barchi-Chung A, Enfield G, et al. Effects of dietary phosphate restriction and phosphate binderson FGF23 levels in CKD. Clin J Am Soc Nephrol 2013;8:1009-18.

25. Shalhoub V, Shatzen EM, Ward SC, et al. FGF23 neutralization improves chronic kidney disease-associated hyperparathyroidism yet increases mortality. J Clin Invest 2012;122:2543-53.

26. Fliser D, Kollerits B, Neyer U, et al. Fibroblast growth factor 23 (FGF23) predicts progression of chronic kidney disease: the Mild to Moderate Kidney Disease (MMKD) Study. J Am Soc Nephrol 2007;18:2600-8.

27. Isakova $\mathrm{T}$, Xie $\mathrm{H}$, Yang $\mathrm{W}$, et al. Fibroblast growth factor 23 and risks of mortality and end-stage renal disease in patients with chronic kidney disease. JAMA 2011;305:2432-9.

28. Khan AM, Chirinos JA, Litt H, Yang W, Rosas SE. FGF-23 and the progression of coronary arterial calcification in patients new to dialysis. Clin J Am Soc Nephrol 2012;7:2017-22.

29. Gutierrez OM, Januzzi JL, Isakova T, et al. Fibroblast growth factor 23 and left ventricular hypertrophy in chronic kidney disease. Circulation 2009;119:2545-52.

30. Mirza MA, Larsson A, Lind L, Larsson TE. Circulating fibroblast growth factor-23 is associated with vascular dysfunction in the community. Atherosclerosis 2009;205:385-90.

31. Faul C, Amaral AP, Oskouei B, et al. FGF23 induces left ventricular hypertrophy. J Clin Invest 2011;121:4393-408.

32. Munoz Mendoza J, Isakova T, Ricardo AC, et al. Fibroblast growth factor 23 and Inflammation in CKD. Clin J Am Soc
Nephrol 2012;7:1155-62.

33. Xie J, Cha SK, An SW, Kuro OM, Birnbaumer L, Huang CL. Cardioprotection by Klotho through downregulation of TRPC6 channels in the mouse heart. Nat Commun 2012;3:1238.

34. Parker BD, Schurgers $L J$, Brandenburg VM, et al. The associations of fibroblast growth factor 23 and uncarboxylated matrix Gla protein with mortality in coronary artery disease: the Heart and Soul Study. Ann Intern Med 2010;152:640-8.

35. Arnlov J, Carlsson AC, Sundstrom J, et al. Higher fibroblast growth factor-23 increases the risk of all cause and cardiovascular mortality in the community. Kidney Int 2013;83:160-6.

36. Dai B, David V, Martin A, et al. A comparative transcriptome analysis identifying FGF23 regulated genes in the kidney of a mouse CKD model. PLoS One 2012;7:e44161.

37. Soriano S, Ojeda R, Rodriguez M, Almaden Y, Martin-Malo A, Aljama P. The effect of phosphate binders, calcium and lanthanum carbonate on FGF23 levels in chronic kidney disease patients. Clin Nephrol 2013;80(1):17-22.

38. Koiwa $\mathrm{F}$, Onoda $\mathrm{N}$, Kato $\mathrm{H}$, et al. Prospective randomized multicenter trial of sevelamer hydrochloride and calcium carbonate for the treatment of hyperphosphatemia in hemodialysis patients in Japan. Ther Apher Dial 2005;9:340-6.

39. Finch JL, Tokumoto $M$, Nakamura $\mathrm{H}$, et al. Effect of paricalcitol and cinacalcet on serum phosphate, FGF-23, and bone in rats with chronic kidney disease. Am J Physiol Renal Physiol 2010; 298:F1315-22.

40. Wetmore JB, Liu S, Krebill R, Menard R, Quarles LD. Effects of cinacalcet and concurrent low-dose vitamin D on FGF23 levels in ESRD. Clin J Am Soc Nephrol 2010;5:110-6.

41. Chertow GM, Block GA, Correa-Rotter R, et al. Effect of cinacalcet on cardiovascular disease in patients undergoing dialysis. $\mathrm{N}$ Engl J Med 2012;367:2482-94.

42. Wohrle S, Henninger $\mathrm{C}$, Bonny $\mathrm{O}$, et al. Pharmacological inhibition of fibroblast growth factor (FGF) receptor signaling ameliorates FGF23-mediated hypophosphatemic rickets. J Bone Miner Res 2013;28:899-911.

43. Aono $Y$, Yamazaki $Y$, Yasutake J, et al. Therapeutic effects of anti-FGF23 antibodies in hypophosphatemic rickets/osteomalacia. J Bone Miner Res 2009;24:1879-88. 